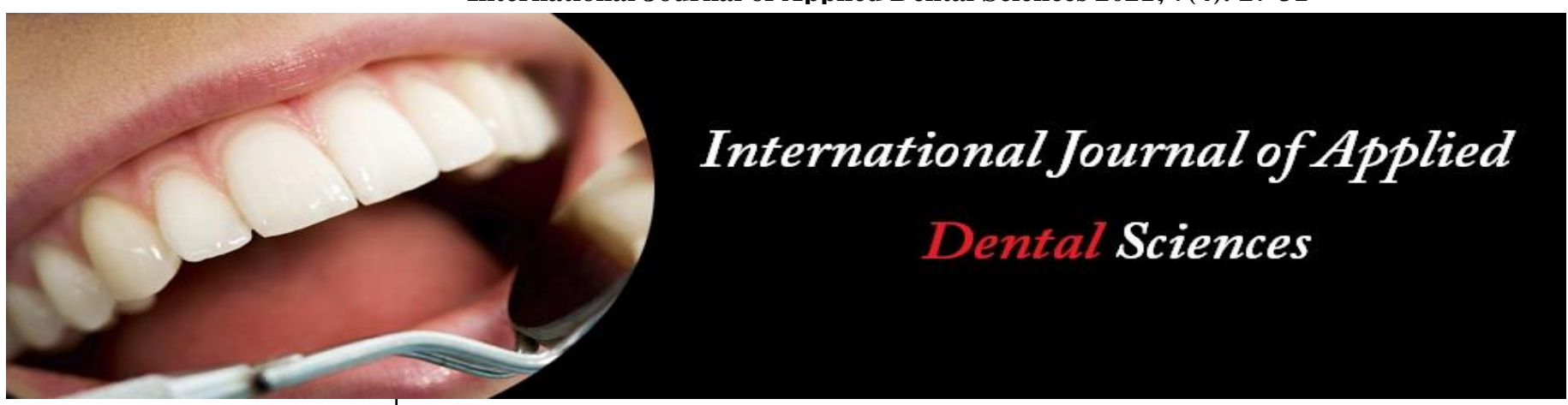

ISSN Print: 2394-7489

ISSN Online: 2394-7497

IJADS 2021; 7(4): 27-31

(C) 2021 IJADS

www.oraljournal.com

Received: 22-08-2021

Accepted: 24-09-2021

Dr. Manpreet Kaur Sandhu MDS (Conservative Dentistry \& Endodontics), Consultant Endodontist, Fortis Hospital, Mohali, Punjab, India
Corresponding Author: Dr. Manpreet Kaur Sandhu MDS (Conservative Dentistry \& Endodontics), Consultant Endodontist, Fortis Hospital, Mohali, Punjab, India

\section{Techniques for Gutta Percha (GP) removal in retreatodontics (Part 1)}

\section{Dr. Manpreet Kaur Sandhu}

DOI: $\underline{\text { https://doi.org/10.22271/oral.2021.v7.i4a.1349 }}$

\section{Abstract}

For successful endodontics, major emphasis is placed on judicious instrumentation, microbial control and complete obturation of the root canal system. During retreatment of previously endodontically treated teeth, one may encounter variety of different materials, which must be removed before these objectives can be achieved. The intent of this article is to present various methods for Gutta Percha removal from the root canal system to aid in re-treatment attempts. Gutta Percha is the most commonly used obturating material, so, therefore this article deals with various techniques available for its removal in retreatment cases.

Keywords: Gutta Percha, hand instruments, retreatment in endodontics, rotary instruments

\section{Introduction}

With improved skill and knowledge of the dentist along with advanced technologies in endodontics there has also been increased necessity for retention of tooth rather than extraction. Patients nowadays are more convinced that dental procedures are painless, manageable and successful. Yet, endodontic failures can transpire at times due to various reasons which can be exasperating both for dentist as well the patient. So, the retreatment comes into play which may entail lengthy treatment periods or sometimes additional visits. The most commonly used obturating material in endodontic treatment is Gutta Percha. Hence, this article emphasizes strictly on various techniques for Gutta Percha removal in Retreatodontics.

\section{Techniques}

1. K-files or H-files

2. Micro debriders or micro openers

3. GP solvents

4. Combination of paper points and GP solvents

5. Rotary instruments

i) Gates glidden drills/ peeso reamers

ii) GPX Gutta Percha remover

iii) GPR System

iv) NiTi rotary instruments

6. Specialized rotary instruments designed for re-treatment

i) Pro Taper universal retreatment instruments

ii) Mtwo retreatment files

iii) R-Endo retreatment files

iv) XP Endo retreatment files

7. Heat transfer devices

i) Heat carrier tips

ii) Ultrasonic tips

8. Soft tissue laser 


\section{K-files or H-files}

These are used to engage mass of Gutta Percha (GP). By application of outward firm pressure mass of GP can be removed. This method is most commonly used in retreatment cases for gross GP removal especially from large canals with poor compaction. This allows files to bypass the obturating material and 'bite' into the mass.

More recently safe ended hand files ${ }^{[1]}$ has been introduced from Mani (Fig. 1a \& 1b)

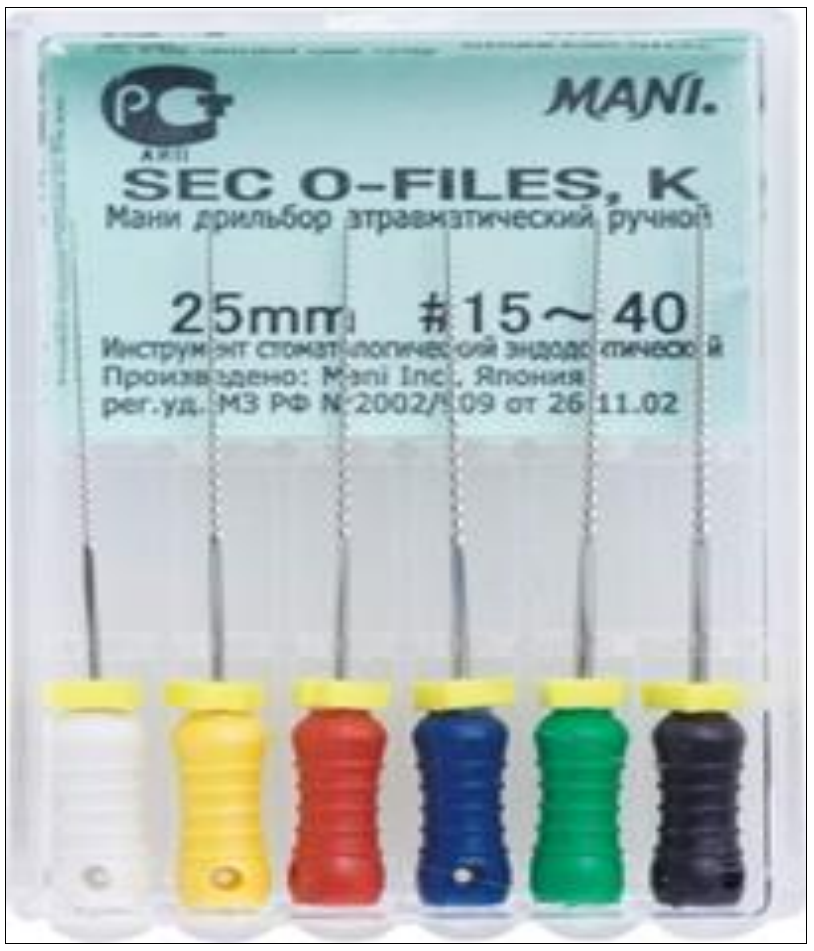

Fig 1a: Safe ended K files (Mani)

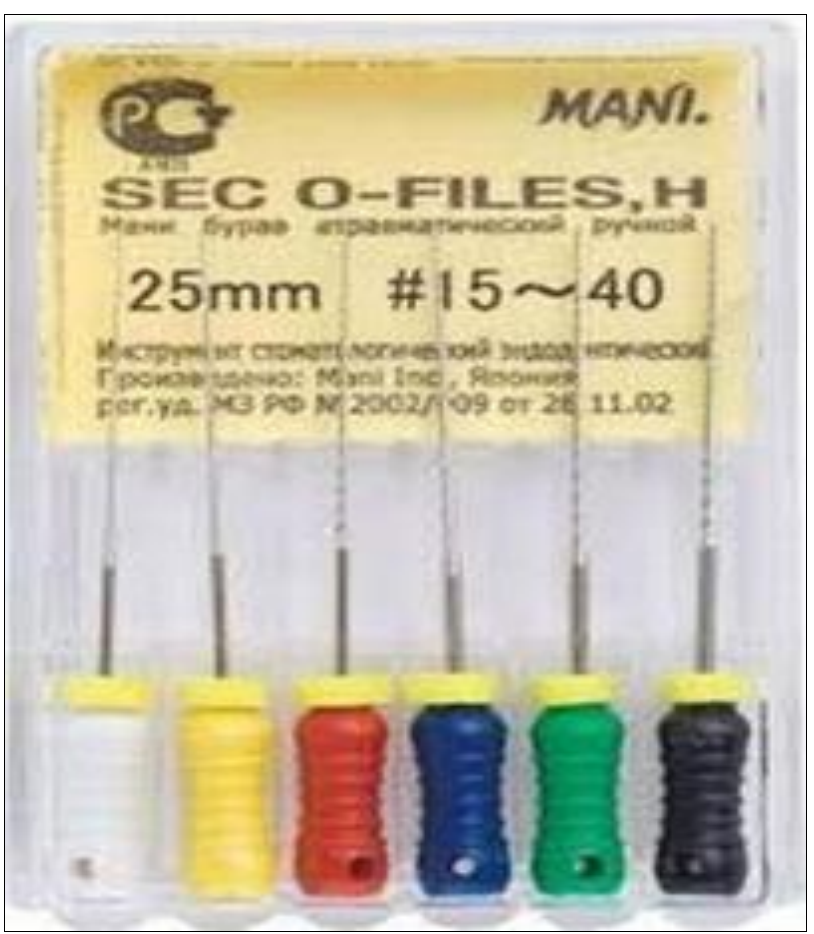

Fig 1b: Safe ended H files (Mani)

\section{Micro debriders or micro openers}

These are similar to K-files and H-files in their usage. These are small files having 90 degree bend at the working end and an attached handle (Fig. 2)
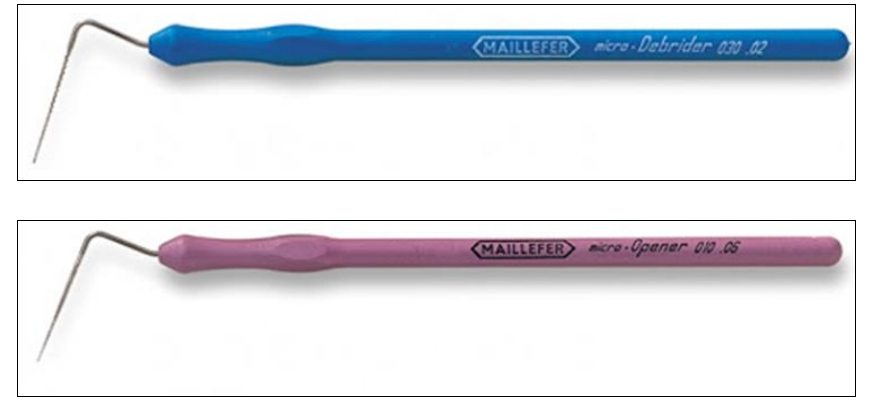

Fig 2: Micro debriders and micro openers (Dentsply Maillefer)

\section{GP solvents}

These include wide array of chemicals like eucalyptol oil, turpentine, chloroform, methyl chloroform, methylene chloride, orange wood oil, tetrahydofuran, xylene, xylol.

Trichloroethylene, cineole, orange oil, Coe Paste Remover, halothane, anise oil, anethole, bergamot oil, terpineol in cineole, chlorobutanol in cineole, methoxyflurane, and diethyl ether have been tried and tested ${ }^{[2]}$.

The eucalyptol oil does not effectively dissolve GP at room temperature and has to be heated to act relatively fast. Hence, its not widely used.

Chloroform is preferred due to volatility, cost, availability, better odor and compatibility with zinc oxide-eugenol-based root canal sealers ${ }^{[2]}$. But due to reported adverse effects on health from chloroform exposure, less hazardous solvents are commonly used ${ }^{[3]}$.

These solvents softens the GP and this softened mass can be easily removed by placing files inside the canals followed by application of firm pressure against the canal walls.

\section{Combination of paper points and GP solvents}

This includes 'wicking technique' wherein solvent is flushed into canals upto the level of pulp chamber to increase maximum interaction of solvent with GP. This is followed by drying it with paper points, which helps in removing the softened GP along with paper points ${ }^{[3,4]}$.

This method is very useful in narrow canals or canals with greater degree of curvature ${ }^{[3]}$.

\section{Rotary instruments}

i) Gates glidden drills/ peeso reamers: These are used for GP removal in coronal and middle third of root canals (Fig. 3a $\& 3 b)$. The non-flexible head and lateral cutting design of these instruments do not allow instrumentation in curved portion of canals. Hence, due to their stiffness and tendency to fracture, they are safer in straight portion of canals in anterior and posterior teeth.

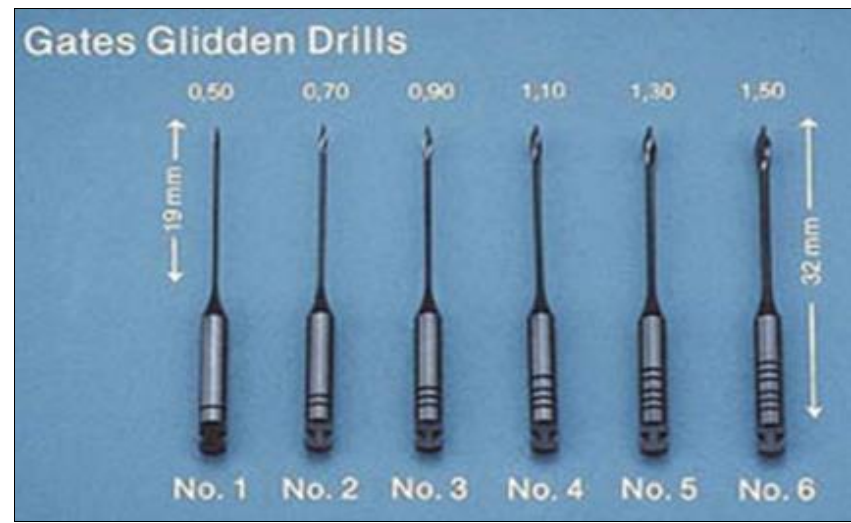

Fig 3a: Gates glidden drills no. 1-6 identified by head design and rings on latch type attachment 


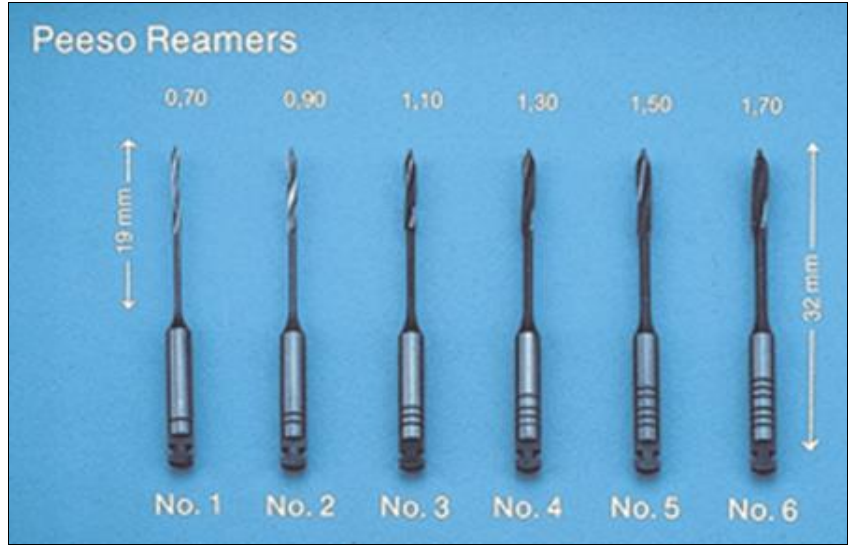

Fig 3b: Peeso reamers no. 1-6 with longer length of flutes on head design and rings on latch type attachment

ii) GPX Gutta Percha remover (Brasseler USA): This is specially designed file used in slow speed handpiece. It plasticizes GP by frictional heat and facilitates its removal from root canal by its $\mathrm{H}$-file like flute design. Stainless steel drills are more effective in coronal and middle third of root canals while Ni-Ti GPX removers can also be used in curved canals (Fig. 4a \& 4b). It has no cutting edges: won't "chew up" Gutta Percha. It has spiral vent through which Gutta Percha is extruded as its plasticized from frictional heat, thus pulled out from the canal wall.

\section{GPXTM - Stainless Steel (6/Pack)}

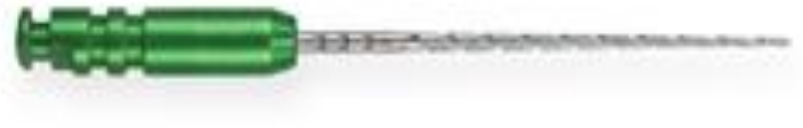

\section{Tip ID Tip Size}

$\begin{array}{ll}\text { Red } & 25 \\ \text { Blue } & 30 \\ \text { Green } & 35 \\ \text { Black } & 40 \\ \text { White } & 45 \\ \text { Yellow } & 50\end{array}$

Speed Setting $2,000-4,000$ RPMs

Fig 4a: GPX Stainless steel



Fig 4b: GPX NiTi

iii) GPR system (Mani): Is a 4-file system (Fig. 5) that is configured into an assorted pack of 4 instruments $(1 \mathrm{~S}, 2 \mathrm{~S}, 3 \mathrm{~N}$, $4 \mathrm{~N})$ and 4 individual sizes i.e. $1 \mathrm{~S}, 2 \mathrm{~S}, 3 \mathrm{~N}$, and $4 \mathrm{~N}$ sizes. The 1 and 2 "S" instruments are stainless steel and the 3 and 4 "N" instruments are nickel titanium. In order of decreasing tip sizes-
1S/\#70 (16 mm, green, .70 mm tip size); for coronal to middle 2S/\#50 (18 mm, yellow, $.50 \mathrm{~mm}$ tip size); for coronal to middle

3N/\#40 (21 mm, black, .40 mm tip size); for middle to apical 4N/\#30 (21mm, blue, .03 mm tip size); for middle to apical All files are teardrop shaped in cross section and .04 tapered. Usually, $1500 \mathrm{rpm}$ is adequate to power the GPR files. 


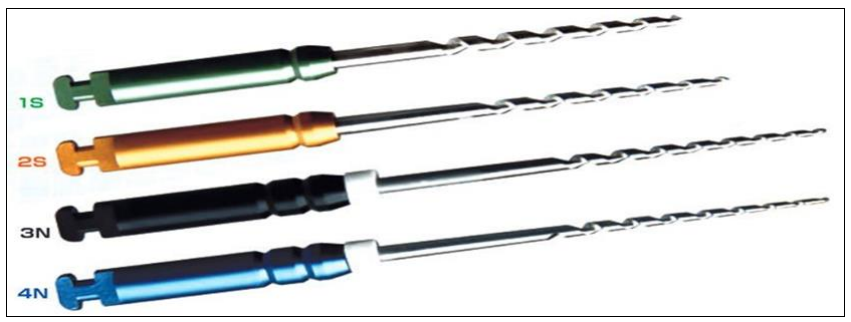

Fig 5: GPR system (Mani)

iv) NiTi rotary instruments: Rotary instruments reaches the working length easily by plasticizing GP through frictional heat and allowing quick GP removal $[5,6,7]$. This should be followed by hand instruments for refining and complete removal.

Recommended rotational speed of these instruments is three to four times more than that for routine cleaning and shaping procedures. Rotary instruments have tendency to fracture if used forcefully through GP mass ${ }^{[3,8,9]}$.

\section{Specialized rotary instruments designed for re- treatment}

i) Pro Taper universal retreatment instruments (Dentsply Maillefer): This retreatment kit comprises of three files designated as D1, D2 and D3 which are marked as one, two and three white rings respectively (Fig. 6). Speed recommended is $500 \mathrm{rpm}$.



Fig 6: Pro Taper universal retreatment instruments (Dentsply Maillefer)

ii) Mtwo retreatment files (VDW, Munich, Germany): It comprises of two files with $\mathrm{S}$-shaped cross section designed to reach the apex (Fig. 7). They carry the advantage of preparing the root canal in an under-prepared canal, simultaneously. Both files have length $21 \mathrm{~mm}$ each.



Fig7: Mtwo Retreatment files (VDW, Munich, Germany)

iii) R-Endo retreatment files (Micro Mega): It comprises of stainless steel $\mathrm{Rm}$ hand file used to break the hard layer of filling material and $4 \mathrm{NiTi}$ instruments in continuous rotation for flaring ( $\mathrm{Re})$ and progressive shaping of the 3 root canal areas (R1, R2 \& R3) in an endo rotary system (Fig. 8a). While $\mathrm{Rm}, \mathrm{Re}, \mathrm{R} 1, \mathrm{R} 2, \mathrm{R} 3$ \& Rs retreatment kit is used with an InGet type contra-angle in a gentle in-and-out motion on canal walls (Fig. 8b). Recommended speed is 300-400 rpm.

$\mathrm{Rm}$ hand file (size 25, 0.04 taper, $17 \mathrm{~mm}$ length, $12 \mathrm{~mm}$ working tip) to relocate the canal orifices,
Re (size 25, 0.12 taper, $15 \mathrm{~mm}$ length, $10 \mathrm{~mm}$ cutting tip) to flare the first few millimeters of the canal, R1, R2 and R3 (size 25, with respective taper of $0.08,0.06,0.04$; length $15 \mathrm{~mm}, 19 \mathrm{~mm}, 23 \mathrm{~mm}$; working tip $8 \mathrm{~mm}, 12 \mathrm{~mm}, 16 \mathrm{~mm}$ ) to remove filling material from coronal, middle and apical third of canals respectively.

Also, an optional finishing file Rs (size 30, 0.04 taper, $15 \mathrm{~mm}$ length, $10 \mathrm{~mm}$ cutting tip) in cases where additional enlargement of apical diameter of canal is required.

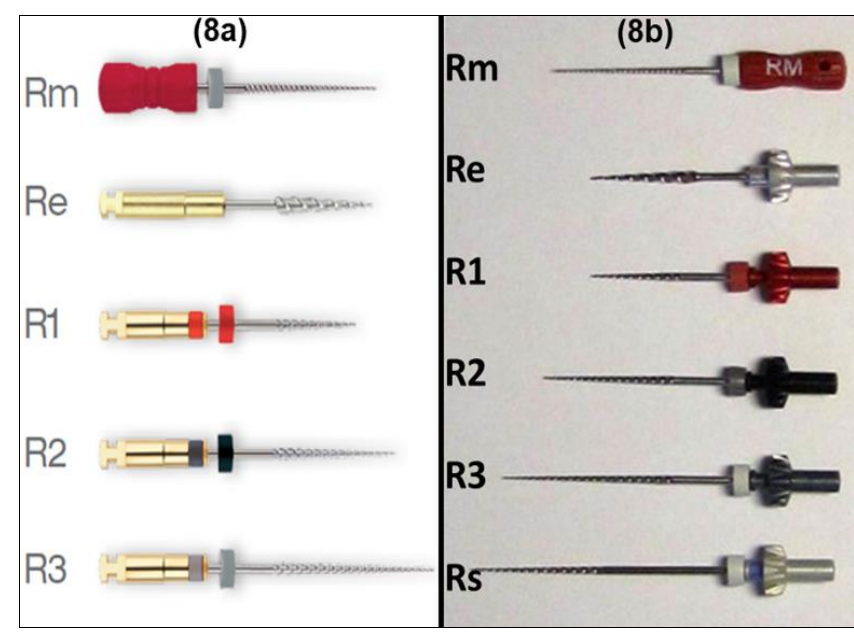

Fig 8a, b: Endo rotary system, In Get system R-Endo retreatment files (Micro Mega) 
(iv) XP Endo retreatment kit (FKG Swiss Endo): Its used in following sequence as DR1(15mm length, orifice opener), XP endo Shaper (size/taper 30/0.04) and XP endo Finisher R (size/taper 30/0.00).

Gently engage DR1 rotating (Fig. 9a) at 800-1000 rpm and $1.5 \mathrm{Ncm}$ torque into the obturation material in order to create a 3-4 mm starting point for the XP-endo Shaper tip (Fig. 9b). Use pecking motion until the XP-endo Shaper's tip engages the Gutta Percha at 1000- $2500 \mathrm{rpm}$. Then use light pressure to help advance the XP-endo Shaper down the canal until working length. Add solvent if needed. Use the XP-endo Shaper for 10-15 additional long gentle strokes to WL and irrigate the canal to eliminate suspended debris. Fix the canal working length on the XP-endo Finisher R (Fig. 9c) at (800$1000 \mathrm{rpm}, 1 \mathrm{Ncm}$ ) and slowly thread the XP-endo Finisher R into the canal. Use the XP-endo Finisher $\mathrm{R}$ for at least 1 minute (longer, if debris is still coming out of the canal), in slow and gentle longitudinal movements to contact the full length of the canal.

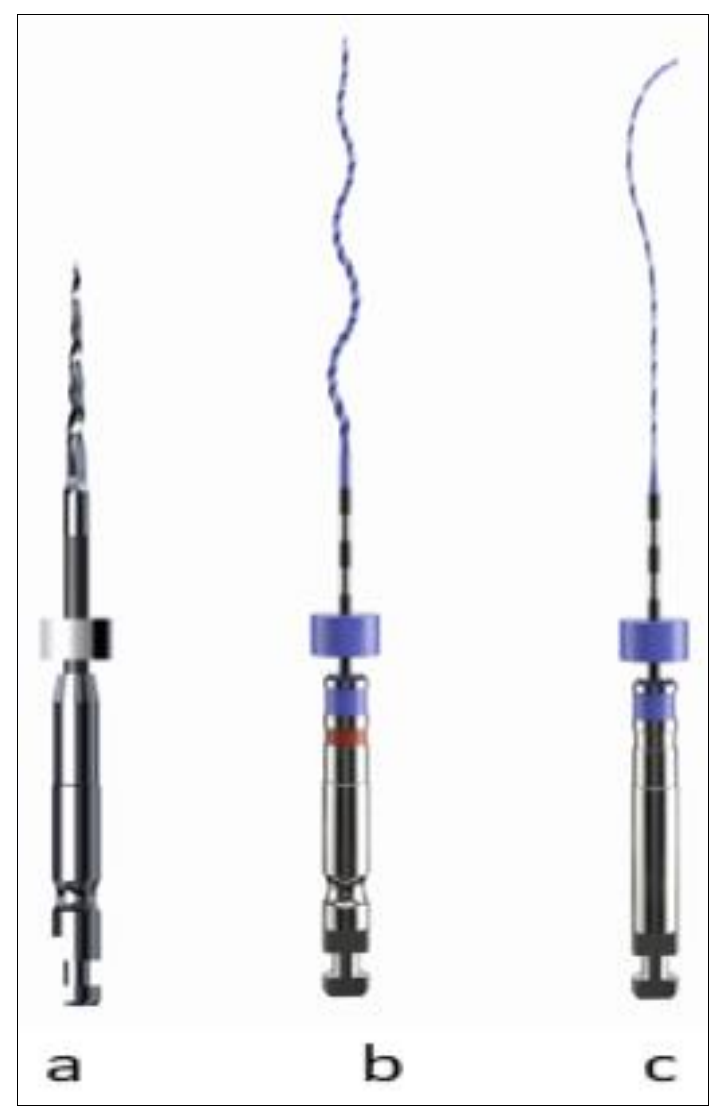

Fig 9a: DR1, 9b - XP endo Shaper \& 9c - XP endo Finisher R

\section{Heat transfer devices}

i) Heat carrier tips: Variety of heat transfer devices like System B, Endotec, Endo Twin E\&Q Mater, Touch N Heat, down Pak etc. are available for warm vertical obturation technique. So, these heat carrier tips can also be used for retrieval of GP mass especially in well prepared canals ${ }^{[3]}$.

ii) Ultrasonic tips - they work in a similar manner as that of heat carrier tips by softening the GP mass. Finer tips of these ultrasonic tips can be used around root canal curvatures also [3].

\section{Soft tissue laser}

The use of $\mathrm{Nd}$ : YAG laser has shown to be capable of softening the GP. Nd: YAG laser at lower settings $(100 \mathrm{~mJ}$, $15 \mathrm{~Hz}, 1.5 \mathrm{~W})$ produces fairly clean root canals, but incomplete GP removal from dentinal walls. At increased power levels $(100 \mathrm{~mJ}, 20 \mathrm{~Hz}, 2 \mathrm{~W})$ the laser seems to be more efficient in cleaning the canal walls ${ }^{[10]}$.

\section{Conclusion}

Nonetheless, it can be concluded that removal of Gutta Percha from root canals is a difficult task which can at times, become lengthy and tiring procedure. Yet, its important to clean the canals effectively and diligently. Every retreatment case should be dealt as a fresh case to achieve the desired success.

\section{References}

1. Mounce R. Using hand files to their full capabilities: A new look at an old yet emerging technology. Autralasian Dent Pract 2013.

2. Wourms DJ, Campbell AD, Hicks ML, Pelleu GB. Alternative solvents to chloroform for gutta-percha removal. Journal of Endodontics 1990;16(5):224-6.

3. Khatavkar R, Hegde V. Current concepts in gutta-percha removal for re-treatment. Dental Tribune 2010, 18.

4. Duncan HF, CHONG BS. Removal of root filling materials. Endodontic Topics 2008;19(1):33-57.

5. Özyürek T, Demiryürek EÖ. Efficacy of different nickeltitanium instruments in removing gutta-percha during root canal retreatment. Journal of endodontics 2016;42(4):646-9.

6. Gu LS, Ling JQ, Wei X, Huang XY. Efficacy of Pro Taper Universal rotary retreatment system for gutta-percha removal from root canals. International Endodontic Journal 2008;41(4):288-95.

7. Kim HC, Kwak SW, Cheung GS, Ko DH, Chung SM, Lee W. Cyclic fatigue and torsional resistance of two new nickel-titanium instruments used in reciprocation motion: Reciproc versus Wave One. Journal of endodontics 2012;38(4):541-4.

8. Sağlam BC, Görgül G. Evaluation of surface alterations in different retreatment nickel-titanium files: AFM and SEM study. Microscopy Research and Technique 2015;78(5):356-62.

9. Arantes WB, Da Silva CM, Lage-Marques JL, Habitante S, Da Rosa LC, De Medeiros JM. SEM analysis of defects and wear on Ni-Ti rotary instruments. Scanning: The Journal of Scanning Microscopies 2014;36(4):411-8.

10. Majori M, Bedini R, Filippini P, Altamura C, Caiazza S. SEM Evaluation of Canal Walls After Nd: YAG Laser Removal of Gutta-Percha. Journal of Oral Laser Applications 2004, 4(4). Soft tissue lasers 